\title{
Calculation of Ramp Response of Lossy Transmission Lines Using Two-port Network Functions
}

\author{
Payam Heydari and Massoud Pedram \\ Dept of EE-Systems \\ University of Southern California \\ Los Angeles, CA 90089 \\ E-mail: \{payam,massoud\}@ zugros.usc.edu
}

\begin{abstract}
In this paper, we present a new analytical approach for computing the ramp response of an RLC interconnect line with a pure capacitive load. The approach is based on the two-port representation of the transmission line and accounts for the output resistance of the driver and the line inductance. The results of our analysis are compared with the results of HSPICE simulations demonstrating the high accuracy of our solution under various values of driver, interconnect, and load impedances.
\end{abstract}

\section{INTRODUCTION}

With the exponential reduction in the feature size, the delays due to interconnections have become the dominating factor in determining the circuit performance. The minimum feature size of devices that can be etched on silicon has decreased from about 8 microns in the late seventies to about 0.18 microns in deep submicron technologies right now. This scaling has resulted in interconnect delay becoming the major bottleneck in today's high speed circuits. Due to aggressive scaling of interconnects even an average length metal line may have significant resistance compared to the driver resistance. Thus the distributed nature of the interconnect must be modeled. Furthermore, the IC operating frequency nears multi-gigahertz implies that the interconnect inductance also needs to be properly modeled.

Recently, some approximation techniques (e.g. AWE and REX) for estimating the time domain response of interconnect structures have been proposed. AWE [1] provides a rapid approximation of general $R L C$ interconnect model and has been successfully applied to analyze on-chip signal propagation. AWE begins with the differential state equations of a lumped LTI (Linear Time-Invariant) circuit and then obtains the Laplace transform solution of the homogeneous equation. This solution is expanded in a McLaurin series, and the time-domain moments are computed from this series and are matched to an approximating function. The approximating function is a linear combination of exponential functions. REX [2] is another approach for rapidly estimating the transient response of lossy transmission line which expands the reciprocal of transfer function of the system. For critical underdamped interconnects, this method provides better result than AWE.

Both of these approaches suffer from inaccuracy especially in high speed integrated circuits. Liao, and Dai [3] proposed using an $S$-parameter based macromodel as a two-port network for modeling the interconnect structures. Another way of obtaining the time domain response of an interconnect line is to solve the Telegrapher's equations. Kahng and Muddu [4] used this approach for a distributed $R C$ interconnection under the ramp excitation. They assumed that a finite number of reflections (namely four) is sufficient for generating a result very close to SPICE simulation. The authors however do not consider the inductive effect of interconnect line in their model and assume that the exciting voltage source has zero valued output resistance.

In this paper, we begin with the two-port model of the transmission line and obtain the time-domain expression of the ramp response for a finite-length $R L C$ lines. The effect of wire inductance and the resistance of CMOS driver of the interconnect is considered in our method. Section 2 summarizes the background knowledge about the Telegrapher's equations. Section 3 presents our analytical method for computing the ramp response of a lossy transmission line. We present our experimental results and concluding remarks in sections 4 and 5, respectively. 


\section{BACKGROUND}

Let's give some definitions and terminology first. A linear circuit belongs to the class of linear time invariant systems. Hence it can be completely characterized by its impulse response. The transient behavior of any linear system is contained in its system function which is the Laplace transform of the impulse response. A uniform transmission line with capacitive load has been depicted in Fig. 1. The transmission line has the property that a signal propagates over the interconnection medium as a wave. The basic electrical parameters of a transmission line are resistance along the line, inductance along the line, conductance shunting the line, and capacitance shunting the line. Fig. 1.b depicts the electrical model of the transmission line.

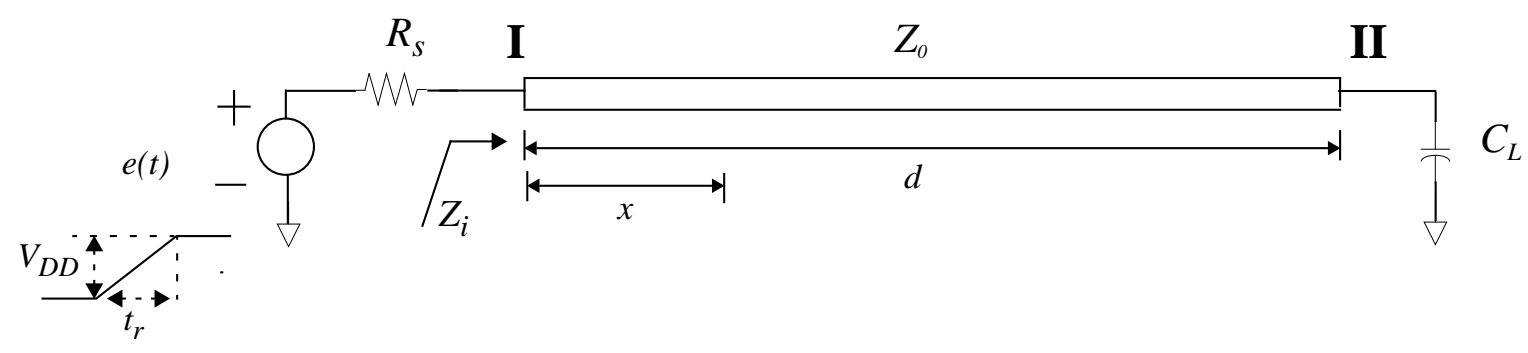

(a)

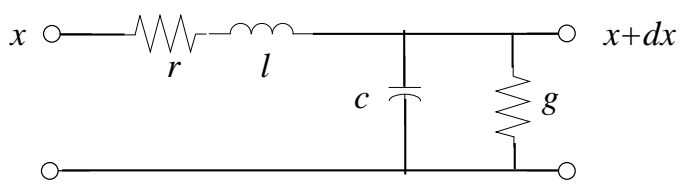

(b)

Fig. 1. Uniform transmission line. (a)Distributed transmission line of length $d$ with a load. (b) Electrical model

Let $r, l, c, g$ be the resistance, inductance, capacitance, conductance values per unit length of a uniform transmission line. The Telegrapher's equations for such a transmission line is [5]:

$$
\begin{aligned}
& \frac{\partial v}{\partial x}=-\left(l \frac{\partial i}{\partial t}+r i\right) \\
& \frac{\partial i}{\partial x}=-\left(c \frac{\partial v}{\partial t}+g v\right)
\end{aligned}
$$

By combining equations (1) and (2), we can come up with the following equation:

$$
\frac{\partial^{2} v(x, t)}{\partial x^{2}}=l c \frac{\partial^{2} v(x, t)}{\partial t^{2}}+(\lg +r c) \frac{\partial v(x, t)}{\partial t}+\operatorname{rg} v(x, t)
$$

Equations (1), (2), (3) are the fundamental relationships governing wave propagation along a uniform transmission line. The shunt conductance is often negligible, hence we set $g=0$. The boundary and initials condition for Eq. (3) are: 
Boundary Condition: $\quad v(0, t)=e(t)$

Initial Conditions: $v\left(x, 0^{-}\right)=0, \frac{\partial v\left(x, 0^{-}\right)}{\partial t}=0$

At each point on the transmission line the voltage or current is the sum of incident and reflected components of the wave. For instance the voltage at point $x$ in Fig. (1) is obtained by the following equation:

$V_{f}(x, t)=$ The first incident wave + The first reflected wave + The second incident wave $+\ldots$

We point out that in the subsequent analysis we model the input voltage as a ramp in the $\left[0, t_{\text {rise }}\right]$ interval and a step function in the $\left[t_{\text {rise }}, \infty\right)$ interval. We obtain the output response for each of the inputs separately. However when obtaining the output response for second part we use the initial condition imposed by the first part of the input waveform.

\section{INTERCONNECT TRANSFER FUNCTION}

Since we are interested in calculating the waveform at the output of the interconnection, we do not go through complicated details of wave reflections through the endpoints of transmission line. Instead we use the two port representation of transmission lines. We use chain parameters for relating the port variable. When two or more interconnect lines are connected in cascade, their $A B C D$ matrices are simply multiplied together ${ }^{1}$ [5], [6].

$$
\left[\begin{array}{l}
V 2 \\
I 2
\end{array}\right]=\left[\begin{array}{ll}
A & B \\
C & D
\end{array}\right]\left[\begin{array}{l}
V 1 \\
I 1
\end{array}\right]=\frac{1}{2}\left[\begin{array}{cc}
\exp (\lambda d)+\exp (-\lambda d) & \frac{\exp (-\lambda d)-\exp (\lambda d)}{Y_{0}(s)} \\
(\exp (-\lambda d)-\exp (\lambda d)) Y_{0}(s) & \exp (\lambda d)+\exp (-\lambda d)
\end{array}\right]\left[\begin{array}{c}
V 1 \\
I 1
\end{array}\right]
$$

where $\lambda=\lambda(s)=\sqrt{s C(s L+R)} \quad, Y_{0}(s)=\sqrt{\frac{s C}{s L+R}}$, and $d$ denotes the length of the transmission line. $L, R$, and $C$ denote the total line inductance, line resistance, and line capacitance, i.e. $L=d l, R=d r$, and $C=d c$.

On the other hand, the voltage at port II, is related to the current at port II by the load capacitor equation (cf. Fig. 1.). Hence the transfer function of the interconnection loaded by a capacitor $C_{L}$ is obtained as:

$$
H(s)=\frac{V_{2}}{V_{1}}=\frac{2}{\left(e^{\lambda d}+e^{-\lambda d}\right)\left(1+Z_{0}(s)\left(C_{L} s\right) \tanh (\lambda d)\right)}
$$

where $Z_{0}(s)=1 / Y_{0}(s)$. The inverse of the first parenthesis in the denominator term of Eq. (6) is a limit summation of a power series. We exploit the related power series of this term since then we will be able to map the series to the time domain with little effort. Consequently, the transfer function is written in the following form:

$$
H(s)=\frac{\sum_{n=1}^{\infty}(-1)^{n+1} e^{-(2 n-1) \lambda d}}{\left(1+Z_{0}(s)\left(C_{L} s\right) \tanh (\lambda d)\right)}
$$

As can be seen from Eq. (7), $\lambda(s)$ and $Z_{0}(s)$ depend upon the square root of frequency variable, $s$. This makes the inverse Laplace transform consist of the error function which does not give a simple formula for the time domain representation of the output waveform. So we extend the McLaurin series of $\lambda(s)$ and $Z_{0}(s)$, and then based on practical values of parameters, truncate the series into the first two terms of the McLaurin series about $s=\infty$. A good approximation for $\lambda(s)$ and $Z_{0}(s)$ is given:

1. Notice that this property is very useful when analyzing the ramp response of a cascade of interconnect segments with different widths. 


$$
\lambda(s)=\sqrt{L C} s+\frac{1}{2} R \sqrt{\frac{C}{L}} \text { and } Z_{0}(s)=\sqrt{\frac{L}{C}}\left(1+\frac{R}{2 L s}\right)
$$

Notice that neglecting resistive term in $Z_{0}(s)$ expression yields the well known characteristic impedance for a lossless transmission line and that the propagation delay of wave through the interconnect media is completely present in the approximation to $\lambda(s)$. Combining Eqs (7) and (8), the transfer function of a lossy transmission line is obtained as:

$$
H(s)=\frac{\sum_{n=1}^{\infty}(-1)^{n+1} e^{-(2 n-1) \frac{R}{2} \sqrt{\frac{C}{L}}} e^{-(2 n-1) \sqrt{L C} s}}{\left(1+Z_{0}(s)\left(C_{L} s\right) \tanh (\lambda d)\right)}
$$

The above approximation for $\lambda(s)$ causes a large change in the DC value of the transfer function. We alleviate this error by adding a gain compensation factor to the transfer function.

To find an effective gain, let $H_{1}(s)$ be defined as:

$$
H_{1}(s)=\frac{1}{\left(1+Z_{0}(s)\left(C_{L} s\right) \tanh (\lambda d)\right)}
$$

The output expression is then composed of the delayed versions of $h_{l}(t)$, the inverse Laplace transform of $H_{1}(s)$ :

$$
v_{\text {out }}(t)=\sum_{n=1}^{\infty}(-1)^{n+1} e^{-(2 n-1) \frac{R}{2} \sqrt{C / L}} h_{1}[t-(2 n-1) T]
$$

where $T$ is the time of flight of the wave. Since $T$ is very small compared to temporal changes of $h_{1}(t)$, we can approximately assume that the delay is negligible, factorize $h_{1}(t)$, and put it outside the summation. We therefore come up with the following equation:

$$
v_{\text {out }}(t) \cong h_{1}(t)\left\{\sum_{n=1}^{\infty}(-1)^{n+1} e^{-(2 n-1) \frac{R}{2} \sqrt{C / L}}\right\}
$$

The limit of the power series in Eq. (12) gives us an idea about the steady-state value of $v_{\text {out }}(t)$ which is interpreted as $V_{\text {out }}(0)$ in the $s$-domain (the final-value theorem [8]). Doing this we obtain:

$$
v_{\text {out }}(t) \cong h_{1}(t) \frac{e^{-\frac{R}{2} \sqrt{C / L}}}{1+e^{-R \sqrt{C / L}}}
$$

The actual steady-state value of $h_{l}(t)$ is one. The error is due to the second term in right hand-side of Eq. (13). Considering the practical values of interconnect parasitics, we can see that $\exp (-R \sqrt{C / L}) \ll 1$. Consequently the compensating gain is set as $\exp ((R / 2) \sqrt{C / L})$. The modified transfer function after taking this multiplicative factor into consideration is written as:

$$
H(s)=H_{1}(s) \cdot \sum_{n=1}^{\infty}(-1)^{n+1} e^{-(n-1) R \sqrt{\frac{C}{L}}} e^{-(2 n-1) \sqrt{L C} s}
$$

$H_{1}(s)$ depends upon the Laplace transform of voltage at port I. Now we do further manipulation to make the analysis more efficient. We propose the following piece-wise linear function as an approximation to $\tanh ($.$) :$

$$
\tanh (\lambda l)=\left\{\begin{array}{cc}
1 & \lambda d \geq 2 \\
0.5(\lambda d) & 0 \leq \lambda d \leq 2
\end{array}\right.
$$

For each of the values of $\tanh ($.$) we will obtain the transfer function and the ramp response of the single lossy$ 
interconnect in the following subsections.

$H(s)$ in Eq. (14) denotes the relation between the voltages at the output port (i.e. port II) and the input port (i.e. port I) of the interconnect line. If we wish to have the relation between the output voltage of the interconnect and the source voltage $e(t)$, we have to consider the voltage division between the driver impedance and the input impedance $Z_{i}(s)$ seen by looking into the interconnect. We know from [6] that $Z_{i}(s)$ is:

$$
Z_{i}(s)=z_{11}(s)-\frac{z_{12} z_{21}}{z_{22}+Z_{L}}
$$

where $z_{11}, z_{22}, z_{21}, z_{12}$ are the two-port open-circuit impedance parameters and $Z_{L}$ is the load impedance. By knowing the chain parameters, any of the other sets of two-port parameters, such as the $z$-parameters, can be computed [6]. Hence the input impedance of the interconnect can be expressed in terms of the parameters of interconnect.

$$
Z_{i}(s)=\frac{\left(e^{\lambda d}-e^{-\lambda d}\right)+Z_{L} Y_{0}\left(e^{\lambda d}+e^{-\lambda d}\right)}{Y_{0}\left[\left(e^{\lambda d}+e^{-\lambda d}\right)+Z_{L} Y_{0}\left(e^{\lambda d}-e^{-\lambda d}\right)\right]}=\frac{Z_{L}\left(1+Z_{0} Y_{L} \tanh (\lambda d)\right)}{1+Z_{L} Y_{0} \tanh (\lambda d)}
$$

We can use a similar piece-wise linear approximation for $\tanh ($.$) which was used in Eq. (15). Using this approxi-$ mation however yields a forth-order source to output transfer function which make the output waveform so complicated. In order to avoid this complexity, we use different approximation as explained next.

Therefore we can ignore the $e^{-\lambda d}$ term in comparison with the $e^{\lambda d}$ term. Now we should show that this approximation does not cause a large amount of error. We can rewrite Eq. (17) as the following form:

$$
Z_{i}(s)=\frac{Z_{L}}{1+Z_{L} Y_{0} \tanh (\lambda d)} \cdot \frac{1}{H_{1}(s)}
$$

Since we are concerned about the magnitude of errors we can write the magnitude of the frequency response of Eq. (18) as explained below:

$$
\left|Z_{i}(j \omega)\right|=\left|\frac{Z_{L}(j \omega)}{1+Z_{L}(j \omega) Y_{0}(j \omega) \tanh (\lambda(j \omega) d)}\right| \cdot \frac{1}{\left|H_{1}(j \omega)\right|} \leq\left|Z_{L}(j \omega)\right| \cdot \frac{1}{\left|H_{1}(j \omega)\right|}
$$

$\left|Z_{L}(j \omega)\right|$ is very small for current high-speed circuits. As frequency increases obviously $\left|Z_{L}(j \omega)\right|$ becomes even smaller. Any error in approximating $H_{l}(s)$ is multiplied by this small value, and henceforth we get a small error even if $\tanh ($.$) is estimated as a unity value over the whole range of \lambda(s)$. In practical cases usually $e^{\lambda d}$ is at least 3 times greater than $e^{-\lambda d}$. For instance using the interconnect parameters for $0.18 \mu$ CMOS technology, and assume a $1 \mathrm{~mm}$ of Metal1 wire, a typical value for $\lambda d$ at $500 \mathrm{MHz}$ clock frequency would be around 1.3 [7]. For global interconnect lines, this value is even larger. This causes $e^{\lambda d}$ to be 13 times larger than $e^{-\lambda d}$. Based on the above approximation, we come up with the following expression for $Z_{i}(s)$ :

$$
Z_{i}(s)=\frac{1}{Y_{0}(s)}=Z_{0}(s)
$$

Consequently the output voltage of the interconnect line is related to source voltage, $e(t)$, by a simple voltage division made by $Z_{i}(s)$ and $R_{s}$.

$$
V_{1}(s)=E(s) \frac{Z_{i}}{Z_{i}+R_{s}}=\frac{Z_{0}}{R_{s}+Z_{0}} E(s)
$$

In the following subsections each of the two cases for approximating $\tanh ($.$) are considered separately. The output$ waveform is obtained for each of two cases. 
CASE I. $\tanh (\lambda d)=1$ :

In this case $H_{1}(s)$ is represented by a first-order rational function of $s$ as follows:

$$
H_{1}(s)=\frac{1}{\sqrt{\frac{L}{C}} C_{L}\left(s+\frac{R}{2 L}+\frac{1}{C_{L}} \sqrt{\frac{C}{L}}\right)}
$$

Comparing Eq. (22) with the actual value of $H_{1}(s)$, again we see that the actual DC value of $H_{1}(s)$ differs from the DC value of the approximated expression of $H_{1}(s)$. This difference will affect the steady state value of the output voltage. To overcome this we can add up a constant multiplicative gain so that the DC value of Eq. (22) becomes unity value. Therefore we will have:

$$
H_{1}(s)=\frac{\frac{R}{2 L}+\frac{1}{C_{L}} \sqrt{\frac{C}{L}}}{\left(s+\frac{R}{2 L}+\frac{1}{C_{L}} \sqrt{\frac{C}{L}}\right)}
$$

As said before we break up the input waveform into two parts: (i). ramp input $e_{r}(t)$ (ii). step input $e_{S}(t)$. Hereafter we use the convention that any voltage variable with index $r$ is related to the ramp input, and any voltage variable with index $s$ is related to the step section of the input. Output response is computed for each of these parts.

Let's consider the first part of the input. From Eq. (21) we are able to obtain the input voltage to transmission line:

$$
V_{r}(s)=\frac{V_{D D}}{t_{\text {rise }}} \frac{\sqrt{\frac{L}{C}}}{\sqrt{\frac{L}{C}}+R_{s}} \cdot \frac{s+\frac{R}{2 L}}{\left(s+\frac{\sqrt{\frac{L}{C}}(R /(2 L))}{\sqrt{\frac{L}{C}}+R_{s}}\right) s^{2}}
$$

We apply partial fraction expansion to $V_{r}(s)$ and then, after obtaining the response to each of the fractional terms, we simply utilize the superposition property to calculate the final value of the output response as follows. Eq. (25) represents the partial fraction expansion of $V_{r}(s)$ :

$$
V_{r}(s)=\frac{V_{D D} / t_{\text {rise }}}{s^{2}}-\frac{R_{s}}{\frac{R}{2 L} \sqrt{\frac{L}{C}}}\left(\frac{1}{s}-\frac{1}{s+\frac{\sqrt{L / C}(R /(2 L))}{\sqrt{L / C}+R_{s}}}\right)
$$

As can be seen from the above equation, three terms are present in the partial fraction expansion of the voltage at port I. We name each of the terms as $V_{r 1}(s), V_{r 2}(s)$, and $V_{r 3}(s)$, respectively. The Laplace transform of the output voltage at port II is composed of the response to each of the three terms. We name each of the output terms as $V_{r 1}{ }^{o}(s)$, $V_{r 2}{ }^{o}(s)$, and $V_{r 3}{ }^{o}(s)$, respectively. We know from signal and system analysis that the Laplace transform of the system response is a product of the system transfer function and the Laplace transform of the input[8]. Consequently we have:

$$
V_{r 1}^{o}(s)=H_{1}(s) V_{r 1}(s)=\frac{V_{D D}}{t_{\text {rise }}} \cdot \frac{\frac{1}{C_{L}} \sqrt{\frac{C}{L}}+\frac{R}{2 L}}{s^{2}\left(s+\frac{1}{C_{L}} \sqrt{\frac{C}{L}}+\frac{R}{2 L}\right)}
$$

Applying partial fraction expansion, then taking inverse Laplace transformation, we come up with the temporal waveform of the output. 


$$
v_{r 1}^{o}(t)=\frac{V_{D D}}{t_{\text {rise }}} r(t)-\left(\frac{V_{D D}}{t_{\text {rise }}} \cdot \frac{1}{\frac{1}{C_{L}} \sqrt{\frac{C}{L}}+\frac{R}{2 L}}\right)\left(1-e^{-t / \tau_{L}}\right) \quad 0 \leq t \leq t_{\text {rise }}
$$

where: $r(t)=t u(t)$ and $1 / \tau_{L}=\frac{1}{C_{L}} \sqrt{\frac{C}{L}}+\frac{R}{2 L}$.

Similarly, we repeat the above steps to obtain the temporal waveforms of $v_{r 2}{ }^{o}(t), v_{r 3}{ }^{o}(t)$ as follows:

$$
\begin{aligned}
& v_{r 2}^{o}(t)=-\left(\frac{V_{D D}}{t_{\text {rise }}} \cdot \frac{R_{s}}{\frac{R}{2 L} \sqrt{\frac{L}{C}}}\right)\left(1-e^{-t / \tau_{L}}\right) \quad 0 \leq t \leq t_{\text {rise }} \\
& v_{r 3}^{o}(t)=\frac{V_{D D}}{t_{\text {rise }}} \cdot \frac{R_{s}}{\frac{R}{2 L} \sqrt{\frac{L}{C}}} \cdot \frac{\left(\left(1 / C_{L}\right) \sqrt{\frac{C}{L}}+R /(2 L)\right)\left(\sqrt{L / C}+R_{s}\right)}{R R_{s} /(2 L)+1 / C_{L}+\left(R_{s} / C_{L}\right) \sqrt{C / L}}\left(e^{-t / \tau_{s}}-e^{-t / \tau_{L}}\right) \quad 0 \leq t \leq t_{\text {rise }}
\end{aligned}
$$

where: $1 / \tau_{s}=\frac{\sqrt{L / C}(R /(2 L))}{\sqrt{L / C}+R_{s}}$

The output voltage is composed of algebraic summation of $v_{r 1}{ }^{o}(t), v_{r 2}{ }^{o}(t), v_{r 3}{ }^{o}(t)$ :

$$
v_{r}^{o}(t)=v_{r 1}^{o}(t)+v_{r 2}^{o}(t)+v_{r 3}^{o}(t) \quad 0 \leq t \leq t_{r i s e}
$$

For the time interval $\left[t_{\text {rise }}, \infty\right)$ the input has a step form, $e_{s}(t)$. The output voltage is made up of the step input and the initial condition imposed by the ramp input. As a result of the continuity condition, the interconnect response to the second part of the input at time $t_{\text {rise }}$ must be equal to the response of the interconnect to the first part at that same time. The same relationship exists for voltages at all other points especially the voltage at the input port of the interconnect, that is,

$$
v_{r}\left(t=t_{\text {rise }}\right)=v_{s}\left(t=t_{\text {rise }}\right)
$$

As defined before, $v_{r}(t)$ is the input voltage of port I when the excitation is in the form of a ramp whereas $v_{s}(t)$ is the input voltage of port I when the excitation is a step function. $v_{r}(t)$ is simply determined by taking the inverse Laplace transformation from Eq. (23).

$$
v_{r}(t)=\frac{V_{D D}}{t_{\text {rise }}} r(t)-\left(\frac{V_{D D}}{t_{\text {rise }}} \cdot \frac{R_{s}}{(R /(2 L)) \sqrt{L / C}}\right)\left(1-e^{-t / \tau_{s}}\right)
$$

$V_{s}(s)$ is determined by substituting $E(s)$ in Eq. (21) with the Laplace transform of the step function. Furthermore it should include an extra term which specifies the response under the initial condition. After taking the inverse Laplace transformation, we obtain the following expression for $v_{r}(t)$ :

$$
v_{s}(t)=V_{0} e^{-t / \tau_{s}}+V_{D D}\left(1-\frac{R_{s}}{R_{s}+\sqrt{L / C}} e^{-t / \tau_{s}}\right)
$$

In Eq.(33) the unknown variable $V_{0}$ is determined by imposing Eq. (31). We can subsequently write: 


$$
v_{s}(t)=\frac{V_{D D}}{t_{\text {rise }}} \cdot \frac{R_{s}}{\frac{R}{2 L} \sqrt{\frac{L}{C}}}\left(1-e^{t_{\text {rise }} / \tau_{s}}\right) e^{-t / \tau_{s}}+V_{D D} \quad t \geq t_{\text {rise }}
$$

We take the same steps as was taken for obtaining the response for interval $\left[0, t_{\text {rise }}\right]$ to derive the response for $\left[t_{\text {rise }}, \infty\right) . v_{s}(t)$ is composed of two terms. One is exponentially rising in time and asymptotically goes toward a constant value, while the other is constant in time. We name $v_{s 1}{ }^{o}(t), v_{s 2}{ }^{o}(t)$ as the system responses to each of the above portions of the $v_{s}(t)$. The interconnect response to each of these signals are:

$$
\begin{aligned}
& v_{s 1}^{o}(t)=V_{D D}\left(1-e^{-t / \tau_{L}}\right) \quad t \geq t_{\text {rise }} \\
& v_{s 2}^{o}(t)=\frac{V_{D D}}{t_{\text {rise }}} \cdot \frac{R_{s}}{\frac{R}{2 L} \sqrt{\frac{L}{C}}}\left(1-e^{t_{\text {rise }} / \tau_{s}}\right) \cdot \frac{\left(\left(1 / C_{L}\right) \sqrt{\frac{C}{L}}+R /(2 L)\right)\left(\sqrt{L / C}+R_{s}\right)}{R R_{s} /(2 L)+1 / C_{L}+\left(R_{s} / C_{L}\right) \sqrt{C / L}}\left(e^{-t / \tau_{s}}-e^{-t / \tau_{L}}\right) \quad t \geq t_{\text {rise }}
\end{aligned}
$$

The final expression of the output voltage is composed of the two functions of Eq. (35), and Eq. (36):

$$
v_{s}^{o}(t)=v_{s 1}^{o}(t)+v_{s 2}^{o}(t) \quad t \geq t_{\text {rise }}
$$

The system response is an algebraic summation of $v_{s}^{o}(t)$ and $v_{r}^{o}(t)$.

CASE II. $\tanh (\lambda d)=0.5(\lambda d)$ :

In this case we expect that the equations become more complicated. Since the order of the transfer function is increased. As you will see this complexity also appears in the output voltage waveform such that the waveform asymptotically goes to its final value with ringing. Similar to the previous case, we change the DC value of the transfer function such that it becomes identical to the DC value of the actual transfer function:

$$
H_{1}(s)=\frac{\omega_{n}^{2}}{s^{2}+2 \alpha s+\omega_{n}^{2}}
$$

where $\omega_{n}^{2}=\frac{R^{2}}{8 L^{2}}+\frac{1}{L C_{L}}$ and $\alpha=\frac{R}{4 L}$.

The denominator has always two complex conjugate poles, hence the response has underdamped behavior. So we observe ringing effect on the output waveform. The methodology for obtaining the output waveform is the same as that used for case I. So we avoid going through the details of computations. For interval $\left[0, t_{\text {rise }}\right]$, the output has composed of three terms according to the following:

$$
v_{r 1}^{o}(t)=\frac{V_{D D}}{t_{\text {rise }}} r(t)-\left(\frac{2 V_{D D}}{t_{\text {rise }}} \cdot \frac{\alpha}{\omega_{n}^{2}}\right) u(t)-\frac{V_{D D}}{t_{\text {rise }}} \cdot \frac{e^{-\alpha t}}{\omega_{d}} \cos \left(\omega_{d} t-\theta\right) \quad 0 \leq t \leq t_{\text {rise }}
$$

where $\omega_{d}=\sqrt{\omega_{n}^{2}-\alpha^{2}}, \theta=\arctan \left(\frac{\alpha^{2}-\omega_{d}^{2}}{2 \alpha \omega_{d}}\right)$.

$$
v_{r 2}^{o}(t)=-\left(\frac{V_{D D}}{t_{\text {rise }}} \cdot \frac{R_{s}}{\frac{R}{2 L} \sqrt{\frac{L}{C}}}\left(1-\frac{\omega_{n}}{\omega_{d}} e^{-\alpha t} \cos \left(\omega_{d} t-\varphi\right)\right)\right) \quad 0 \leq t \leq t_{\text {rise }}
$$


where $\varphi=\arctan \left(\frac{\alpha}{\omega_{d}}\right)$.

$$
v_{r 3}^{o}(t)=\frac{V_{D D}}{t_{\text {rise }}} \cdot \frac{R_{s}}{\frac{R}{2 L} \sqrt{\frac{L}{C}}}\left(\frac{\omega_{n}{ }^{2}}{\left[\left(1 / \tau_{s}\right)^{2}-2 \alpha\left(1 / \tau_{s}\right)+\omega_{n}^{2}\right.} e^{-t / \tau_{s}}-\frac{\omega_{n}^{2}}{\omega_{d}{\sqrt{\omega_{n}^{2}+\left(1 / \tau_{s}\right)^{2}-2 \alpha\left(1 / \tau_{s}\right)}}^{-\alpha t} \cos \left(\omega_{d} t-\xi\right)}\right) \quad 0 \leq t \leq t_{\text {rise }}
$$

where $\xi=\arctan \left(\frac{\alpha-\left(1 / \tau_{s}\right)}{\omega_{d}}\right)$

The output voltage is again stated as an algebraic summation of $v_{r 1}^{o}(t), v_{r 2}^{o}(t), v_{r 3}^{o}(t)$. The response for the step portion of the input is determined by the same methodology which was used for the step portion of the input in case I. Similar to case I, the output is composed of two terms, $v_{s 1}{ }^{o}(t), v_{s 2}{ }^{o}(t)$, due to the two separable parts of the input:

$$
\begin{aligned}
& v_{s 1}^{o}(t)=V_{D D}\left(1-\frac{\omega_{n}}{\omega_{d}} e^{-\alpha t} \cos \left(\omega_{d} t-\varphi\right)\right) \quad t \geq t_{\text {rise }} \\
& v_{s 2}^{o}(t)=V_{s 0}\left(\frac{\omega_{n}{ }^{2}}{\left[\left(1 / \tau_{s}\right)^{2}-2 \alpha\left(1 / \tau_{s}\right)+\omega_{n}{ }^{2}\right]} e^{-t / \tau_{s}}-\frac{\omega_{n}^{2}}{\omega_{d} \sqrt{\omega_{n}^{2}+\left(1 / \tau_{s}\right)^{2}-2 \alpha\left(1 / \tau_{s}\right)}} e^{-\alpha t} \cos \left(\omega_{d} t-\xi\right)\right) \quad t \geq t_{\text {rise }}
\end{aligned}
$$

where $V_{s 0}=\frac{V_{D D}}{t_{\text {rise }}} \cdot \frac{R_{s}}{\frac{R}{2 L} \sqrt{\frac{L}{C}}}\left(1-e^{t_{\text {rise }} / \tau_{s}}\right)$. Again $v_{s}^{o}(t)=v_{s 1}^{o}(t)+v_{s 2}^{o}(t) \quad t \geq t_{\text {rise }}$.

Finally the system response is $v_{s}^{o}(t)+v_{r}^{o}(t)$.

From Eq. (11), the final value of the output is a summation of delayed versions of the computed output voltage. Since the attenuation factor is large, we only consider a few terms of Eq. (11) to calculate the final value of the voltage. Experiments have shown that four terms are sufficient to produce high accuracy. With this approximation the final value of the output voltage will be:

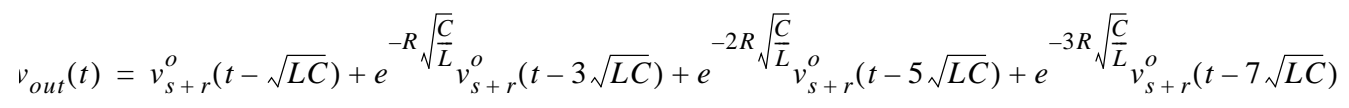

$v_{s+r}^{o}(t)$ means the output voltage which consists of a response to the step portion and a response to the ramp portion. i.e., $v_{s+r}^{o}(t)=v_{s}^{o}(t)+v_{r}^{o}(t)$.

\section{EXPERIMENTAL RESULTS}

We examined several examples with different values of interconnect length and technology, and load and source impedances. Among these experiments four representative examples are reported. Figs. (2) and (4) show the ramp response of two lossy interconnects with $\lambda$ less than two. The analysis of case II is used for these two figures. Fig. (2.a) depicts the output waveform obtained by our analysis whereas Fig. (2.b) shows the output waveform of the same configuration by HSPICE simulation. Similarly Figs (4.a) and (4.b) show the output response of another interconnect obtained by our analysis and HSPICE simulation respectively. Figs. (3) and (5) show the ramp responses of lossy interconnects with $\lambda$ greater than two. The analysis of case I is used for these two cases. Notice that one important feature is kept in our analysis. The output starts from zero with slope equal to zero (differentiability at the starting point). This confirms the correctness of our method.

Table 1. compares the rise-time of the waveforms derived by our analysis with those derived by HSPICE simulation for a number of different transmission lines. Rows 1 through 4 correspond to the transmission lines depicted in 
Fig. 2 through Fig. 5. The remaining rows represent new data points. In table 1. WD represents the width of each conductor, $H T$ is the height of conductor, and $T H$ is thickness of the conductor.

Table 1: Comparison between rise-times of our analysis and HSPICE

\begin{tabular}{|l|l|l|l|l|l|l|l|l|l|l|l|}
\hline $\begin{array}{l}\text { WD } \\
(\mu \mathrm{m})\end{array}$ & $\begin{array}{l}\boldsymbol{H T} \\
(\mu \mathrm{m})\end{array}$ & $\begin{array}{c}\boldsymbol{T H} \\
(\mu \mathrm{m})\end{array}$ & $\begin{array}{l}\boldsymbol{R}_{\boldsymbol{s}} \\
\mathrm{k} \Omega\end{array}$ & $\begin{array}{c}\boldsymbol{C}_{\boldsymbol{L}} \\
\mathrm{pF}\end{array}$ & $\begin{array}{c}\boldsymbol{R} \\
\Omega\end{array}$ & $\begin{array}{c}\boldsymbol{C} \\
\mathrm{pF}\end{array}$ & $\begin{array}{c}\boldsymbol{L} \\
\mathrm{nH}\end{array}$ & $\begin{array}{c}\boldsymbol{d} \\
\mathrm{mm}\end{array}$ & $\begin{array}{c}\boldsymbol{t}_{\text {rise }} \\
(\mathrm{ours}) \\
\mathrm{nsec}\end{array}$ & $\begin{array}{c}\boldsymbol{t}_{\text {rise }} \\
(\mathrm{HSPICE}) \\
\mathrm{nsec}\end{array}$ & error \\
\hline \hline 3 & 2 & 0.8 & 2 & 0.03 & 70.8 & 1 & 3.05 & 10 & 0.49 & 0.65 & $24.6 \%$ \\
\hline 0.2 & 2.5 & 0.25 & 2 & 0.01 & 680 & 0.0736 & 1.45 & 2 & 0.3 & 0.39 & $23 \%$ \\
\hline 0.3 & 2.5 & 0.25 & 2 & 0.01 & 227 & 0.043 & 0.635 & 1 & 0.23 & 0.22 & $4.35 \%$ \\
\hline 0.25 & 2.5 & 0.25 & 2 & 0.01 & 540 & 0.077 & 1.4 & 2 & 0.38 & 0.4 & $5 \%$ \\
\hline 0.2 & 3 & 1 & 2 & 0.01 & 170 & 0.068 & 1.29 & 2 & 0.45 & 0.4 & $11 \%$ \\
\hline 0.6 & 2 & 0.5 & 2 & 0.01 & 113 & 0.1053 & 1.035 & 2 & 0.74 & 0.63 & $14.9 \%$ \\
\hline 0.6 & 2 & 0.1 & 2 & 0.01 & 567 & 0.0965 & 1.21 & 2 & 0.46 & 0.5 & $8 \%$ \\
\hline 0.25 & 1.0 & 0.25 & 2 & 0.01 & 544 & 0.1 & 1.07 & 2 & 0.5 & 0.55 & $9.1 \%$ \\
\hline 0.25 & 1.75 & 0.25 & 2 & 0.05 & 544 & 0.086 & 1.26 & 2 & 0.57 & 0.69 & $17.4 \%$ \\
\hline
\end{tabular}

\section{CONCLUSION}

In this paper we proposed a new method for obtaining the analytical expression for the ramp response of a lossy interconnect. The inductive effects of the wire line, and most importantly the output resistance of the wire driver were considered in our analysis. We started with the two-port representation of the transmission line. Among various two-port parameters the chain matrix was selected. This kind of two-port matrix allows us to obtain the two port matrix of any number of cascade connections of different wires with different wire sizes very easily by simply multiplying their two-port chian matrices. We then obtained the ramp response of the system by doing some further simplification. The results show that this method is able to obtain the ramp response of the lossy interconnect with small error. 


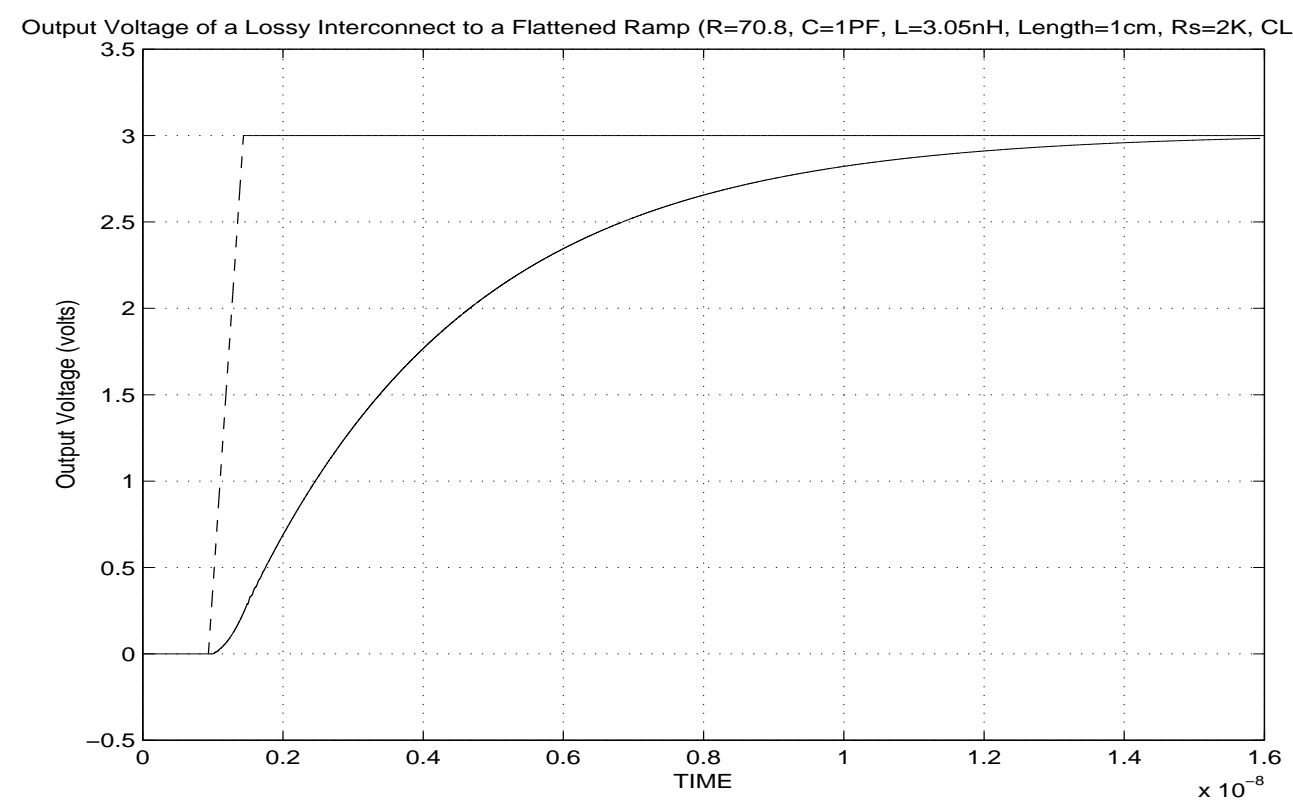

(a)

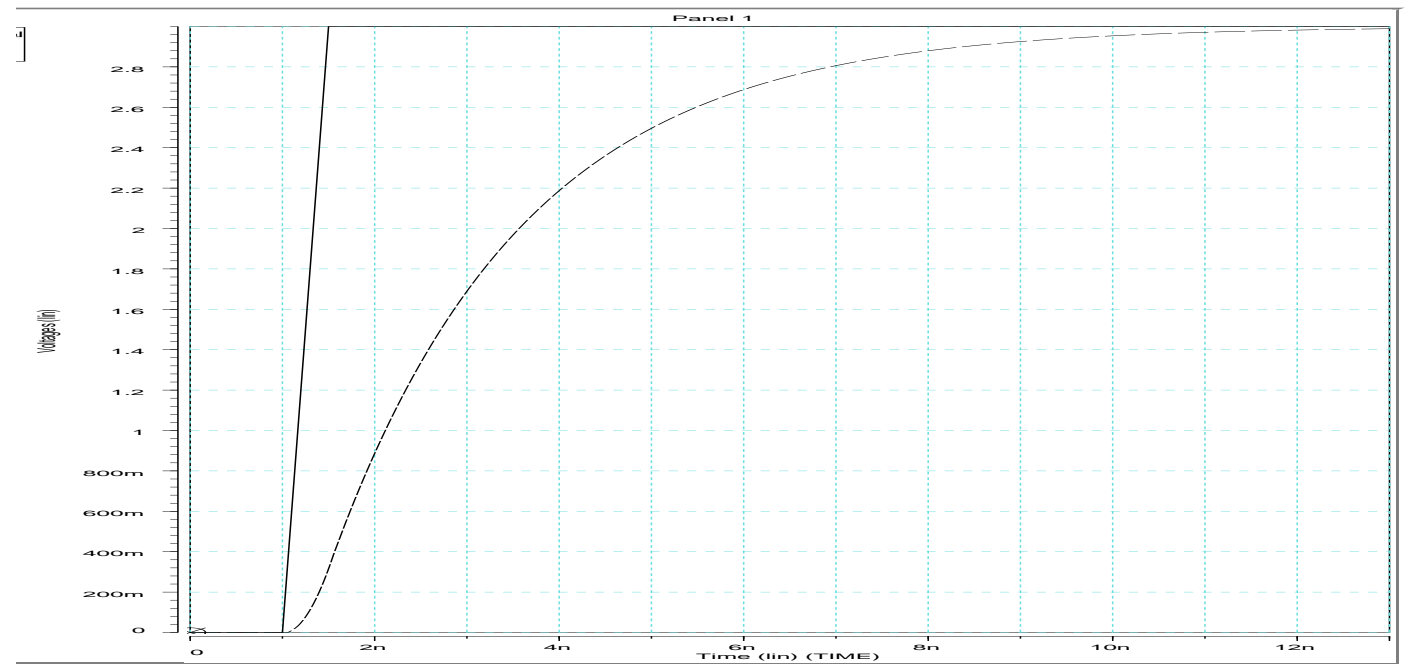

(b)

Fig. (2). Ramp response of a lossy interconnect (Length=1 $\mathrm{cm}, R=70.8, C=1 \mathrm{PF}, L=3.05 \mathrm{nH}$ ) excited by a ramp input with $R_{S}=2 \mathrm{~K}$ as the source resistance, and $C_{L}=0.03 \mathrm{PF}$ as the load capacitance. (a) The result obtained by our method. (b) The result obtained from HSPICE simulation. 


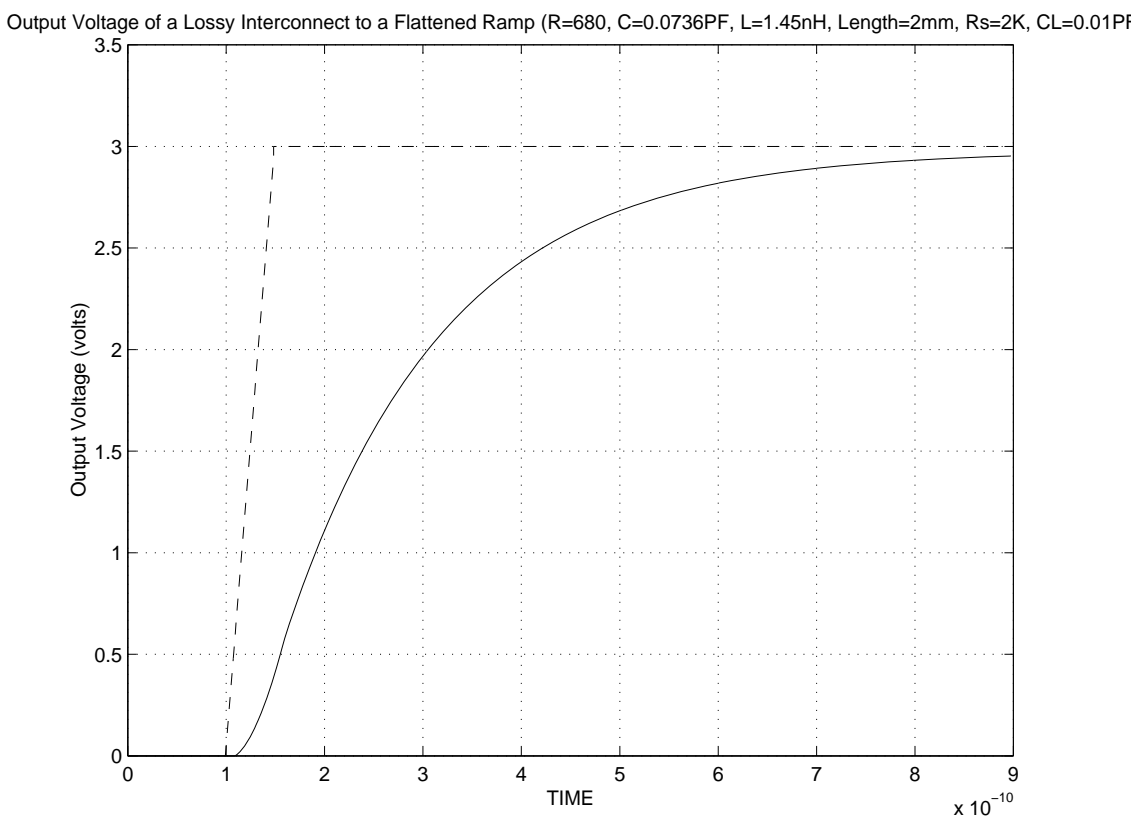

(a)

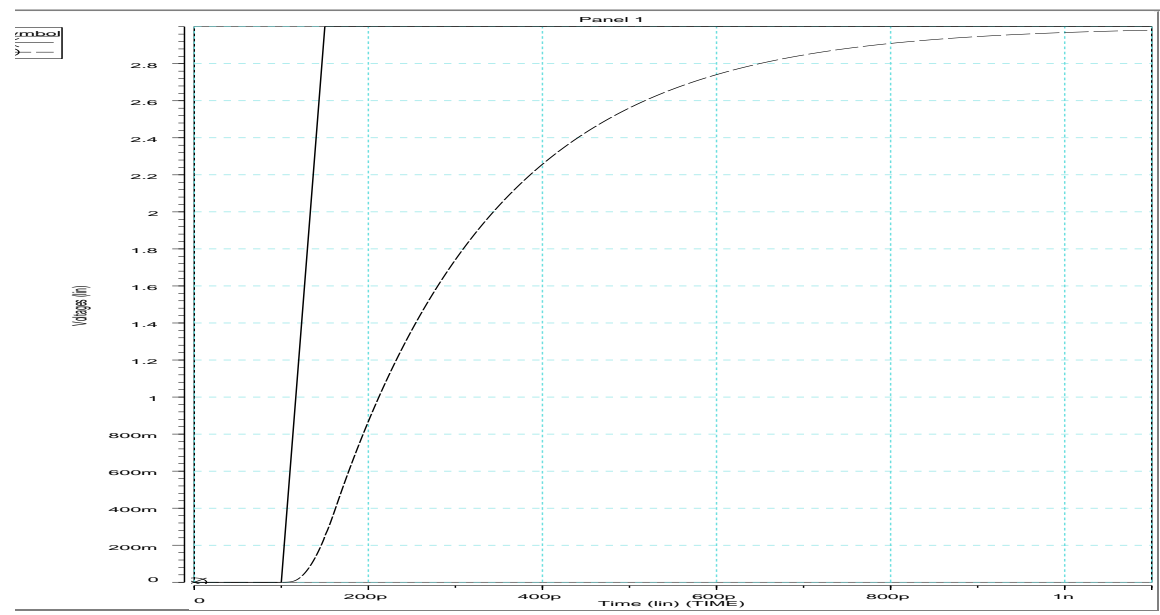

(b)

Fig. (3). Ramp response of a lossy interconnect (Length $=2 \mathrm{~mm}, R=680, C=0.0736 \mathrm{PF}, L=1.45 \mathrm{nH}$ ) excited by a ramp input with $R_{S}=2 \mathrm{~K}$ as the source resistance, and $C_{L}=0.01 \mathrm{PF}$ as the load capacitance. (a) The result obtained by our method. (b) the result obtained from HSPICE simulation. 


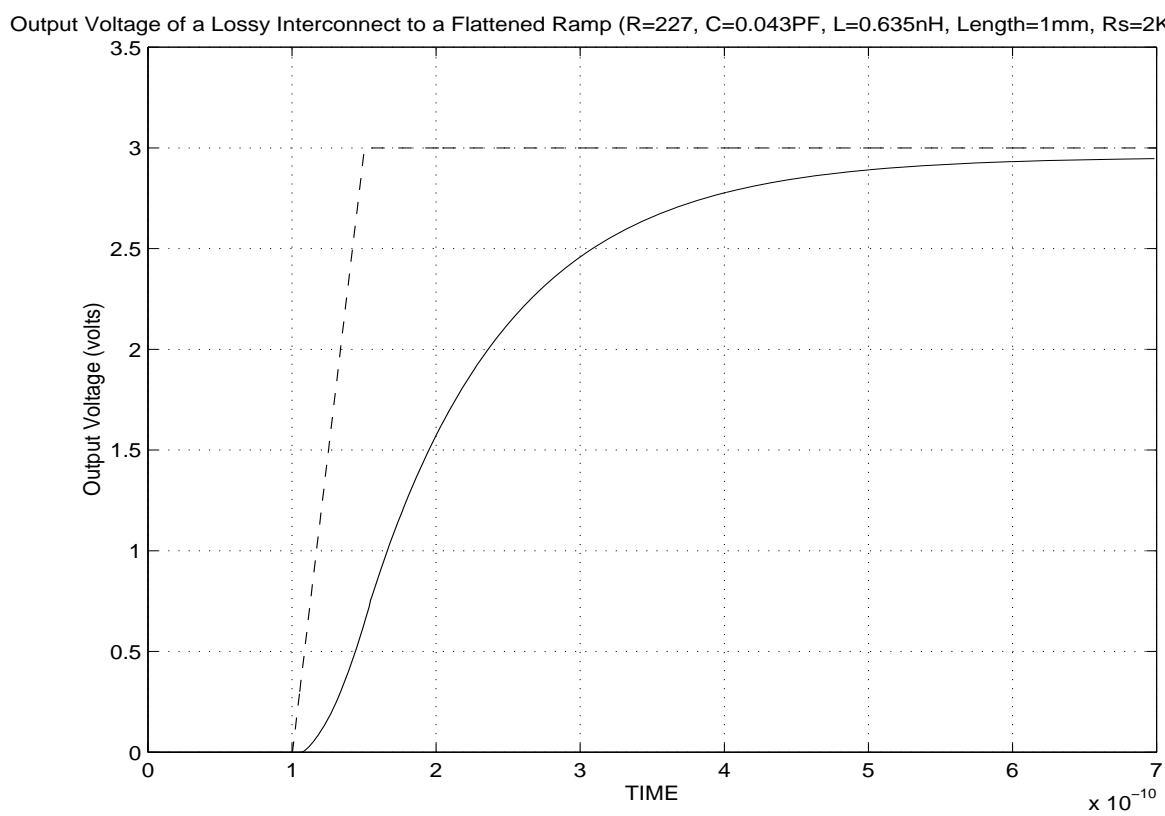

(a)

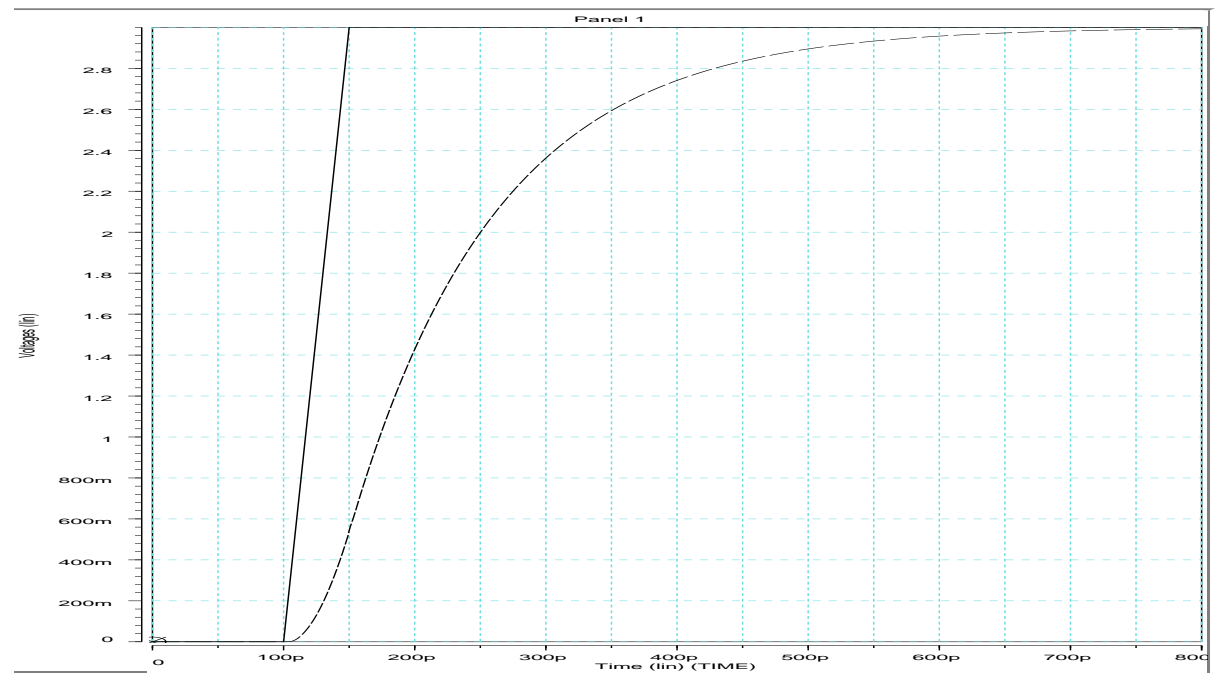

(b)

Fig. (4). Ramp response of a lossy interconnect (Length $=1 \mathrm{~mm}, R=227, C=0.043 \mathrm{PF}, L=0.635 \mathrm{nH}$ ) excited by a ramp input with $R_{S}=2 \mathrm{~K}$ as the source resistance, and $C_{L}=0.01 \mathrm{PF}$ as the load capacitance. (a) The result obtained by our method. (b) the result obtained from HSPICE simulation. 


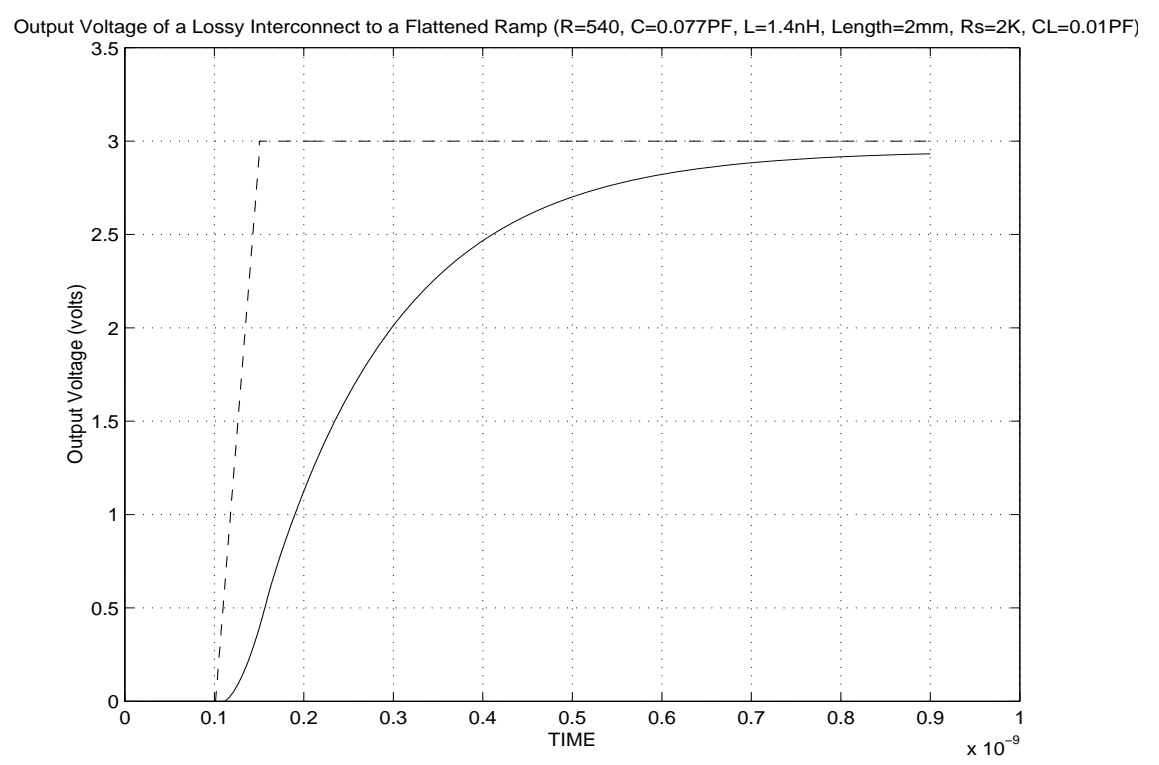

(a)

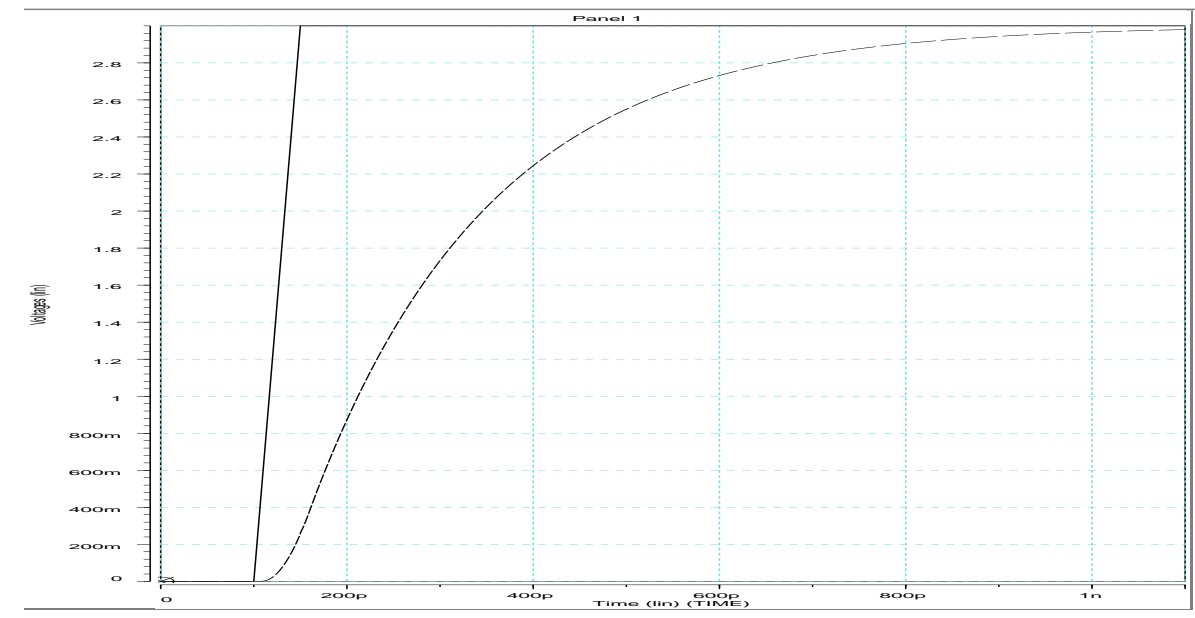

(b)

Fig. (5). Ramp response of a lossy interconnect (Length $=2 \mathrm{~mm}, R=540, C=0.077 \mathrm{PF}, L=1.4 \mathrm{nH}$ ) excited by a ramp input with $R_{s}=2 \mathrm{~K}$ as the source resistance, and $C_{L}=0.01 \mathrm{PF}$ as the load capacitance. (a) The results obtained by our method. (b) the result obtained from HSPICE simulation. 


\section{REFERENCES}

[1] L. T. Pillage and R. A. Rohrer, "Asymptotic Waveform Evaluation for Timing Analysis", IEEE Trans. Comp. Aided Design, vol. 9, pp. 352-366, Apr. 1990.

[2] M. Sriram and S. M. Kang, "Efficient Approximation of the Time Domain Response of Lossy Coupled Transmission Line Trees", IEEE Trans. on Computer-Aided Design of Integrated Circuits and Systems, vol. 14, No. 8, Aug. 1995.

[3] H. Liao, W. Dai, R. Wang, and F. Y. Chang, "S-Parameter Based Macro Model of Distributed-Lumped Networks Using Exponentially Decayed of Polynomial Function", Proc. of the 30th ACM/IEEE Design Automation Conf., pp. 726-731, June 1993.

[4] A. B. Kahng and S. Muddu, "Analysis of RC Interconnections Under Ramp Input", ACM Trans. on Design Automation of Electronic Systems, Jan. 1997.

[5] S. Lin and E. S. Kuh, "Transient Simulation of Lossy Interconnect", Proc. of the 29th ACM/IEEE Design Automation Conf., pp. 81-86, June 1993.

[6] N. Balabanian, T. A. Bickart, Electrical Network Theory, John Wiley \& Sons, INC, Sec. 3.3-3.4, 1969.

[7] J. Cong, Z. Pan, H. He, C.-K. Koh, K.-Y. Khoo, "Interconnect Design for Deep Submicron ICs", Proc. Int. Conf. on Computer Aided Design, pp. 478-485, 1997.

[8] A. V. Oppenheim, A. S. Willsky, H. Nawab, Signals and Systems, 2nd edition, Prentice-Hall, 1996. 